Planetary Systems in the Universe - Observation, Formation and Evolution

Proceedings IAU Symposium No. 202, (c)2004 IAU

Alan Penny, Pawel Artymowicz, Anne-Marie Lagrange, \& Sara Russell, eds.

\title{
Mid-IR Spectroscopy of the Debris Disks in the TW Hydrae Association
}

\author{
Michael L. Sitko \\ Department of Physics, University of Cincinnati, Cincinnati $\mathrm{OH}$ \\ 45221-0011, USA
}

David K. Lynch and Ray W. Russell

The Aerospace Corporation, M2/266, Los Angeles CA 90009-2957, USA

Carol A. Grady

NOAO, Code 681, NASA Goddard Space Flight Center, Greenbelt MD 20771, USA

\begin{abstract}
Spectroscopic observation between 3-14 $\mu \mathrm{m}$ of 3 stars in the TW Hya Association were obtained using the Aerospace Corporation Broadband Array Spectrograph System (BASS) on the NASA IRTF telescope. The targets observed were TW Hya, HR 4796A, and HD 98800. Both of the late-type stars, TW Hya and HD 98800, exhibit a strong $10 \mu \mathrm{m}$ silicate emission band. The strong emission indicates the presence of small dust grains close to the star, at angular distances not currently accessible to coronagraphic techniques. The spectral structure due to crystalline material that is present in some young earlytype stars (HD 163296, HD 31648, etc.) is not apparent in these two objects. For HR $4796 \mathrm{~A}$, the dust emission is weak.
\end{abstract}

\section{Introduction}

The TW Hydrae Association is the nearest region of recent star formation. With an age of approximately $10 \mathrm{Myr}$, it has the potential of providing an important evolutionary link between the young pre-main sequence stars in active starforming clouds, such as Taurus-Auriga, and more mature stellar systems, such as the Vega-like stars. Infrared observations of the stars in TWA indicate the presence of circumstellar dust in many cases, and in a few objects, imaging data confirms the presence of a disk geometry.

As part of an on-going program to investigate the dust characteristics in pre-main sequence disk systems, we obtained mid-IR (3-13 $\mu \mathrm{m})$ spectra of 3 of the brightest members of TWA using the Aerospace Corporation Broadband Array Spectrograph System (BASS) on the NASA IRTF telescope. The targets observed were HD 98800, HR 4796A, and TW Hya itself. 


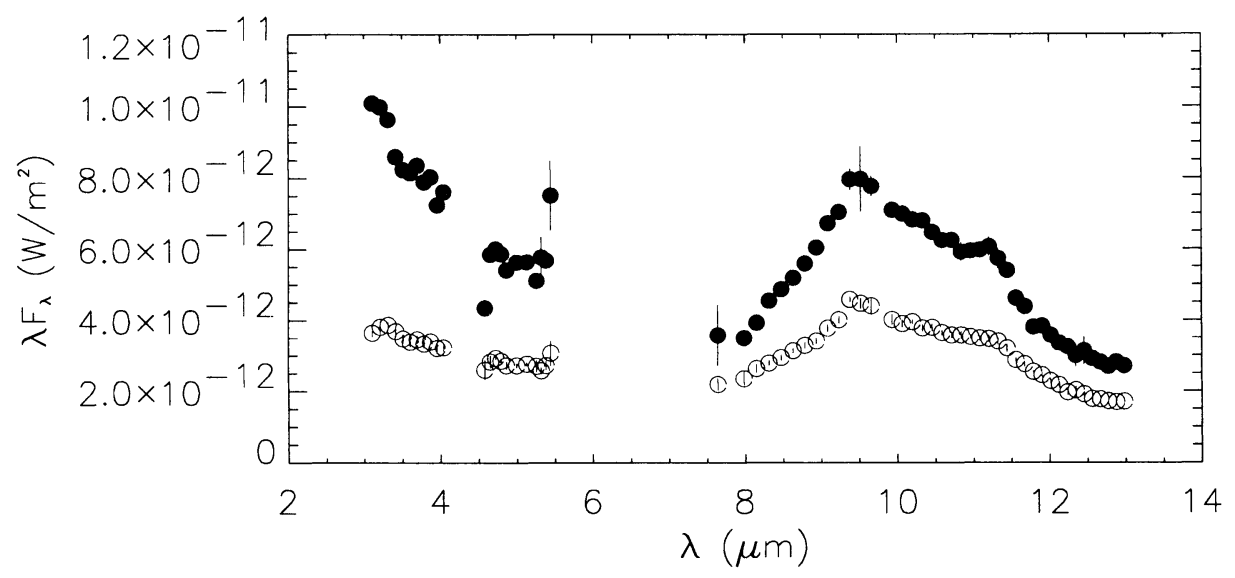

Figure 1. The observed flux of HD 163296 (filled circles) and HD 31648 (open circles). The feature at $11.3 \mu \mathrm{m}$ is due to crystalline olivine.

\section{Results}

In many intermediate-age (4-6 Myr) systems, such as the Herbig Ae/Be stars HD 31648 and HD 163296 (see Figure 1), the silicates have achieved a noticeable degree of crystallinity, as evidenced by the presence of a feature at $11.2-11.3 \mu \mathrm{m}$ due to crystalline olivine (Sitko et al. 1999). While the presence of such a feature has not been conclusively identified with any particular physical or evolutionary process, it may require some degree of annealing and large-scale mixing in the disk material. It is most prominent in older systems, but not all such systems exhibit the feature. What secondary parameters (companion stars, planets, or ??) govern its presence requires further investigation.

The spectrum of HD 98800 (observed by us but also reported by Sylvester \& Skinner 1996) is shown in Figure 2. It exhibits an emission feature indicative of small silicate grains. However, there is no indication of the crystalline olivine feature present in stars such as HD 31648 and HD 163296.

Similarly, TW Hya also possesses a strong silicate band, but again appears to lack the well-defined band structure indicative of a significant degree of crystallinity. The emission bands are similar to those recently observed in $\mathrm{T}$ Tauri stars in the Chamaeleon I dark cloud (Natta et al. 2000).

By contrast with HD 98800 and TW Hya, HR 4796A shows only weak emission above photospheric levels (see Sitko et al. 2000 for a more detailed discussion). This suggests that the disk has been swept nearly clean of material out to the inner edge of the dust ring at $70 \mathrm{AU}$ detected by NICMOS. Unfortunately, the data on HR $4796 \mathrm{~A}$ is of insufficient quality to make any statements concerning its mineralogy or degree of crystallinity. 


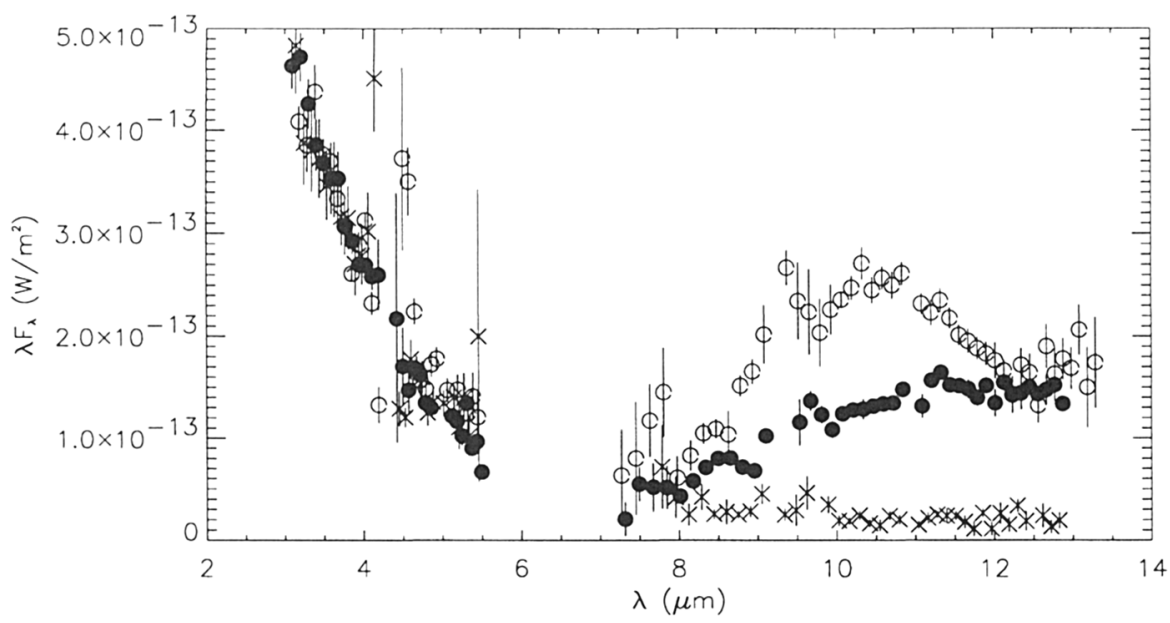

Figure 2. The observed flux of HD 98800 (filled circles), TW Hya (open circles), and HR 4796A ('x' symbols) compared directly. In this figure, the flux of HD 98800 has been scaled down by a factor of 3.5, and that of HR $4796 \mathrm{~A}$ by a factor of 4.5 .

\section{Conclusions}

It is apparent from Figure 2 that the strength of the silicate feature in these three stars is very different from one another. Because these three stars are coeval and presumably formed from the same general mix of material, the strength of this feature must be very sensitive to the detailed evolution of each system. While it is expected that the strength of the feature would decrease with time as the mean grain size increases, the chronological age of the system may be a poor predictor of the mean grain size in any individual system.

Acknowledgments. This work was supported by the URC and Physics Department of the University of Cincinnati (MLS), the Aerospace Corporation IRD program (DKL and RWR). Support for CAG was provided by HST STIS GTO funding through support to the National Optical Astronomy Observatories, in response to NASA A/O OSSA-4-84 through the Hubble Space Telescope Project at Goddard Space Flight Center. NOAO is operated by the Association of Universities for Research in Astronomy (AURA), Inc. under cooperative agreement with the National Science Foundation.

\section{References}

Natta, A., Meyer, M.R., and Beckwith, S.V.W. 2000, ApJ, 534, 838

Sitko, M.L., et al. 1999, ApJ, 510, 408

Sitko, M.L., Lynch, D.K., \& Russell, R.W. 2000, AJ, 120, 2609

Sylvester, R.J., \& Skinner, C.J. 1996, MNRAS, 283, 457 